\title{
Bubble nucleation and growth in fluids
}

\author{
Xavier Frank ${ }^{1}$, Nicolas Dietrich ${ }^{1}$, Jing $\mathrm{Wu}^{2}$, Renaud Barraud ${ }^{1} \&$ Huai Z. $\mathrm{Li}^{1 *}$
}

${ }^{1}$ Laboratoire des Sciences du Génie Chimique, CNRS-ENSIC-INPL, 1 rue Grandville, BP 20451, 54001 Nancy Cedex, France Huai-Zhi.Li@ensic.inpl-nancy.fr

${ }^{2}$ Department of Environmental Science \& Engineering, Tsinghua University, Beijing 100084, P.R. China

Keywords: Bubble, Nucleation, Growth, Non-Newtonian fluids, Visualisation, Micro-PIV

\section{ABSTRACT}

The present paper reports an original study which for the most part is predominantly experimental, investigates the nucleation and growth of $\mathrm{CO}_{2}$ bubbles in non-Newtonian and Newtonian fluids that were initially supersaturated under different pressures. Quantitative information by means of two cameras reveals that at an immobile nucleation site the bubble grows rapidly followed by a linear increase. After reaching a critical size, the bubble detaches from the stagnant site to rise in liquids with an exponential temporary increase for both the diameter and distance. A simple physical reasoning was proposed to qualitatively explain these observed phenomena. Recently, the growth rate and flow fields around a $\mathrm{CO}_{2}$ micro-bubble were measured in a microdevice by a microParticle Image Velocimetry in water. This information at microscale gives new insight into the complex mechanism of bubble nucleation and growth in fluids and could help to develop a rigorous theoretical modelling and numerical simulation such as the Lattice Boltzmann approach. 


\section{INTRODUCTION}

In numerous industrial applications such as polymer devolatilisation process (Yang et al., 1997), generation of biogas bubbles by anaerobic sludge granules in a bioreactor (Wu et al., 2006), polymeric foam production during blow molding (Amon \& Denson, 1984), silicon oil purification (Wienecke et al., 2005), elaboration of microcellular plastics (Xu et al., 2005), carbonated drinks (Liger-Belair et al., 2000; Barker et al., 2002), etc., the mechanism related to the nucleation and subsequent growth of bubbles should be fully understood for engineering analysis of processes (Chhabra, 1992). Similar knowledge is also required in other disciplines like decompression sickness (Kungle \& Beckman, 1983), gas-driven eruption including explosive volcanic eruptions driven by the exsolution of $\mathrm{H}_{2} \mathrm{O}$ from a magma such as the 79 A.D. eruption of Vesuvius or by the exsolution of the $\mathrm{CO}_{2}$ from water like the eruption of the Lake Nyos in 1986 (Zhang, 1998).

The classical nucleation theory assumes that bubble nucleation is initiated by the formation of the critical size nuclei with required energy from the thermodynamical point of view (Abraham, 1974; Jones et al. 1999). After the nucleation, the bubble growth is a complex process involving interactions between mass, momentum and heat transfer and is governed by many characteristic parameters. Usually, the classical equations governing these interactions are numerically resolved as a bubble formation problem (Street et al., 1971; Patel 1980; Shafi \& Flumerfelt, 1997). Until now, there is still very few experimental investigation in the literature. During the growth process, existing works are often limited to an immobile bubble at a stagnant point in liquid. In an industrial devolatilisation installation, the elimination of gas phase is realised by the ascension of bubbles towards a complete separation from liquid. Due to the inherent complex nature of the dynamical coupling between the hydrodynamic and mass transfer aspects, the fundamental mechanisms related to the nucleation and growth of bubbles at microscale in viscous and non-Newtonian fluids remain 
unclear up to day.

The present work aims at investigating the nucleation and growth of $\mathrm{CO}_{2}$ bubbles in both nonNewtonian and Newtonian fluids that are initially supersaturated under different pressures. We examine the nucleation and growth of bubbles not only at a stagnant point in liquids, but also the dynamical growth process during the ascension after the detachment from the nucleation site. Quantitative information concerning these different growth steps along with fine and local measurements at microscale by a micro-Particle Image Velocimetry (micro-PIV) could lead to an increased understanding of these complex phenomena and to improvements of our ability to perform a more rigorous modelling and numerical simulation. In particular, the Lattice Boltzmann (LB) approach that we developed successfully for multiphase flows (Frank \& Li, 2005; Frank et al. 2006; Frank \& Li, 2006) could be a hopeful alternative to classical simulations.

\section{EXPERIMENTAL}

The experimental facilities include a $0.20 \times 0.20 \mathrm{~m}$ square glass visualisation chamber of thin depth $(0.01 \mathrm{~m})$ to avoid too many bubbles in front of the objective of the camera (Fig. 1). At the bottom of the chamber a cylindrical tube of bored wall was used to inject $\mathrm{CO} 2$ gas in the liquid. It was possible to change the initial saturation in liquids by applying various relative pressures of $\mathrm{CO}_{2}$. The whole setup was installed on a mechanical vibrating system so that the dissolution of gas was accelerated to reach a desired saturation degree according to the applied gas pressure. The $\mathrm{CO}_{2}$ was employed as volatile component owning to its adequate dissolution in various liquids. After stopping the vibration of the visualisation chamber, an electronic valve was commanded by a PC to insure a sudden opening to provoke a depressurisation. The nucleation took place in the liquid due to the supersaturation of $\mathrm{CO}_{2}$ under atmospheric conditions and was followed by the growth of 
bubbles. It is worthy noting that both the homogeneous and heterogeneous nucleation was observed respectively in the bulk liquid and at chamber's wall. In the present study, emphasis was given to the homogeneous nucleation. Moreover, the supersaturation degree within the explored experimental conditions ensured the nucleation of limited number of bubbles so that the interactions between bubbles like coalescence did not exist. The investigation was then focused on a single bubble in the bulk liquid in two distinguished steps. Firstly at the beginning of the nucleation, a fixed high speed camera with a great magnification lens was employed to visualise locally a small growing nuclei that stayed still. After reaching a critical size according to fluid's nature and pursuing continuously the growth, the bubble began to leave the nucleation site towards the free surface. A second camera equipped with a less magnification lens was necessary to follow the ascension phase in the liquid over a wide field. Both the size and position of a bubble were determined by the image analysis.

Four non-Newtonian fluids were used in this work: $1 \%$ and $2 \%$ (wt) carboxymethylcellulose $(\mathrm{CMC})$ in water, $0.5 \%(\mathrm{wt})$ polyacrylamide $(\mathrm{PAAm})$ in water, and $0.5 \%(\mathrm{wt})$ xanthan in water. A Rheometrics Fluid Spectrometer RFS II (Rheometrics Inc. USA) was employed to measure the rheological properties of these solutions which behaved as shear-thinning fluids. The CMC and Xanthan solutions were inelastic with no measurable normal forces whereas the PAAm solution was elastic. In the range of shear rates corresponding to the bubble formation and moving in this study $\left(\dot{\gamma}=1-30 \mathrm{~s}^{-1}\right)$, the power law model could fit the rheological behaviour of these fluids:

$$
\tau=K \dot{\gamma}^{n}
$$

where $K$ is the consistency and $n$ the flow index. Their values for the above mentioned fluids are gathered in Table 1.

The growth of an immobile bubble at a nucleation site was firstly studied in these different fluids under various applied relative pressure to control the initial $\mathrm{CO}_{2}$ saturation. The variation of the 
bubble diameter in time is shown in Fig. 2. For a given fluid, the bubble size increases with the applied pressure: a higher initial supersaturation degree produces bigger bubbles. Qualitatively, a more important concentration gradient results in an efficient mass transfer for a fast growth of bubbles' size. In these viscous non-Newtonian fluids, the bubble growth at a nucleation site begins by a very fast period during a short time interval about $2 \mathrm{~s}$, followed by a linear increase until the departure of the bubble. Clearly, the slope of the linear growth is proportional to the initial applied pressure.

When the critical size depending on the fluid's rheology is reached, typically around 1 to $2 \mathrm{~mm}$, the bubble begins the departure from the nucleation site and the ascension takes place in the liquid. A second camera was necessary to follow the displacement of the bubble with a less magnification lens to cover a larger observation field. On the contrary, the image resolution was also affected for the accurate determination of the bubble size. In this case, the main parameter to be measured was then the rising distance from the nucleation site (Fig. 3). The time origin was determined when an observable motion of the bubble began in fluid. Globally, the rising distance increases exponentially with time whatever the experimental conditions may be. The relationship between the rise dynamics, the fluid rheology and the initial pressure doesn't seem to be straightforward. For example, the slope indicates a dependence on the initial relative pressure in $2 \% \mathrm{CMC}$ whereas this isn't the case for $1 \%$ CMC. Further experiments are still required to clarify this point.

Similar exponential increase was roughly observed for the bubble size during the ascension. However, the bubble diameter was difficult to be measured accurately as the second camera covered a large visualisation field. As an indication, some results are given in Fig. 4. 


\section{SIMPLE PHYSICAL INTERPRETATION}

Within the framework of a simplified film model with $\delta$ the film thickness around the bubble, $D$ the diffusion coefficient of $\mathrm{CO}_{2}$ in the liquid, $C$ the concentration of $\mathrm{CO}_{2}$ in the bulk liquid and $C^{*}$ the concentration of $\mathrm{CO}_{2}$ at the bubble interface on liquid side, the gas diffusion flux into the bubble is then

$$
\varphi=\frac{D}{\delta}\left(C-C^{*}\right) S=\frac{D}{\delta}\left(C-C^{*}\right)\left(\pi d_{B}^{2}\right)
$$

The bubble continues to growth as long as the thermodynamical equilibrium isn't reached between the liquid phase and the gas-liquid interface. As a first approach, the uniform pressure inside the bubble and the Henry's law are supposed. With $P_{0}$ as the atmospheric pressure, the Laplace's law gives the pressure inside the bubble: $P_{B}=P_{0}+\frac{2 \sigma}{R}$. This leads then to a concentration at the interface on the liquid side: $C^{*}=\frac{1}{H}\left(P_{0}+\frac{2 \sigma}{R}\right)$. Far from the bubble, the dissolved $\mathrm{CO}_{2}$ concentration $C$ should be superior to $C^{*}$ so that the bubble can growth due to the mass transfer across the bubble interface. Always according to the Henry's law, it could be possible to express this supersaturation by means of an over pressure in the liquid $\Delta P: C=\frac{1}{H}\left(P_{0}+\Delta P\right)$. The mass transfer flux is computed as follows:

$$
\varphi=\frac{D}{\delta H}\left(\Delta P-\frac{2 \sigma}{R}\right)\left(\pi d_{B}^{2}\right)
$$

The experimental curve shown in Fig. 2 for an immobile bubble at the nucleation site has two different growth steps: a rapid growth of bubble diameter followed by a linear increase in time before the departure. This first rapid growth period, typically around $2 \mathrm{~s}$, is nearly independent on 
the fluid's rheology. If the film thickness $\delta$ doesn't change much, the first rapid increase step could be mainly explained by both a high initial value of local supersaturation $\Delta P$ and a significant decrease of the surface tension term due to the size increase $\frac{2 \sigma}{R}$ towards an asymptotically negligible value (Eq. 3).

After $2 \mathrm{~s}$, the establishment of a linear growth implies a certain equilibrium between the gas entering the bubble and mass transfer flux by diffusion from the bulk liquid. The experimental visualisation confirms a spherical shape of bubbles not only at a nucleation site but also during the ascension. The volume of a bubble is then: $V=\frac{\pi}{6} d_{B}^{3}$. The temporary volume variation can be expressed as $\frac{d V}{d t}=\frac{\pi}{2} d_{B}^{2} \frac{d\left(d_{B}\right)}{d t}=\frac{\pi}{2} a d_{B}^{2}$. As the increase rate of the bubble's diameter $a=\frac{d\left(d_{B}\right)}{d t}$ is a constant for the second growth step at the nucleation site as shown in Fig. 2, the mass transfer flow towards the bubble is then linearly proportional to the volume increase rate: $\varphi \sim \frac{d V}{d t}$, a permanent mass transfer regime. However, the attribution of a predominant role is still difficult among three coupling parameters involved in Eq. 3: the film thickness $\delta$, surface tension $\frac{2 \sigma}{R}$ and local supersaturation term $\Delta P$. A plausible assumption could be a constant film thickness, a negligible surface tension when the bubble size is sufficiently great and then a relative constant local supersaturation $\Delta P$. There could be an equilibrium between the mass transfer to the bubble and diffusion flux from the bulk liquid. However, this supersaturation term was difficult to be quantified due to the pressurized chamber used. Some tentative with a probe implemented at the chamber's wall was made by means of $\mathrm{pH}$ measurements in liquids under various applied gas pressure. The accuracy was not sufficient enough to give a satisfactory quantification. Further efforts are still required for measuring this parameter, in particular before the depressurisation. 
To explain the exponential increase for both the bubble's diameter growth and rising distance after the detachment from a nucleation site, an analytical approach was developed for the shear-thinning fluids such as CMC and Xanthan solutions.

The different forces acting on a bubble are mainly:

-the buoyancy: $\vec{F}_{a}=-\left(\rho_{L}-\rho_{G}\right) V \vec{g} \cong-\rho_{L} V \vec{g}$,

-the viscous drag: $\vec{F}_{t}=-\frac{1}{2} \rho_{L} C_{d} \frac{\pi d_{B}^{2}}{4} u^{2} \frac{\vec{u}}{\|\vec{u}\|}$ with the drag coefficient $C_{d}=\frac{16}{\mathrm{Re}}$, generalised

Reynolds number $\operatorname{Re}=\frac{\rho_{L} d_{B} u}{\mu}$ and apparent viscosity $\mu=K \dot{\gamma}^{n-1}=K\left(\frac{u}{d_{B}}\right)^{n-1}$,

-the inertial force due to not only the rising acceleration but also the volume increase: $\frac{d(m \vec{u})}{d t}=\frac{11}{16} \rho_{L}\left(\frac{d V}{d t} \vec{u}+V \frac{d \vec{u}}{d t}\right)$ with a classical added mass of kind of Milne-Thomson $m=\left(\rho_{G}+\frac{11}{16} \rho_{L}\right) V \cong \frac{11}{16} \rho_{L} V$ (Milne-Thomson, 1960; Li et al., 2002),

-the bubble having a spherical shape, the resulting surface tension force in the vertical direction is then zero.

The force balance is as follows:

$$
\frac{d \vec{u}}{d t}=\frac{16}{11 \rho_{L} V}\left(\vec{F}_{a}+\vec{F}_{t}\right)-\frac{1}{V} \frac{d V}{d t} \vec{u}
$$

This is an ordinary differential equation. However, the relationship between the bubble volume $V$ and the volume variation rate $d V / d t$ isn't straightforward and cannot be derived by an analytical approach to our best knowledge. From the experimental data concerning the temporary variation of respectively bubble's diameter and rising distance after the detachment from a nucleation site, it 
could be possible to examine the relationship between these two experimentally measured parameters. For example, the exponential diameter growth curve shown in Fig. 4 gives the following numerical fitting in $1 \% \mathrm{CMC}$ with $P=2$ bars:

$$
\frac{d\left(d_{B}\right)}{d t}=0.68 d_{B}
$$

This is equivalent to the relationship in form of the bubble volume:

$$
\frac{d V}{d t}=\lambda V
$$

where the parameter $\lambda=3 \times 0.68=2.04 \mathrm{~s}^{-1}$.

Eqs (4-6) can then be numerically integrated by a $4^{\text {th }}$ Runge-Kutta method to compute the rising distance with respect to the nucleation site. Fig. 5 shows a satisfactory agreement between the prediction of this simple model and the experimental measurements. This indicates that an exponential growth of the bubble volume (or diameter) leads consecutively to an exponential increase of the rising distance. Both parameters are intrinsically coupled indeed. In another words, the force balance is mainly governed by two principal forces that are buoyancy and drag. Although the bubble growth does introduce some inertial effects, they aren't dominant. Based on this consideration, the asymptotic rise velocity can be obtained by simplifying Eq. 4 :

$$
\begin{aligned}
& \rho_{L} g V-\frac{1}{2} \rho_{L} C_{d} \frac{\pi d_{B}^{2}}{4} u^{2}=0 \\
& u=\left(\frac{1}{12} \frac{\rho_{L} g}{K}\right)^{\frac{1}{n}} d_{B}^{\frac{n+1}{n}}
\end{aligned}
$$

This analytical expression confirms clearly that it is logical to obtain an exponential increase of the rising distance with an exponential growing bubble. Of course, this conclusion is based on the experimental observation of an exponential bubble diameter growth. Back to the above-mentioned 
film assumption for the mass transfer around a bubble, Eq. 3 contains a parameter related to hydrodynamic conditions: the film thickness $\delta$. When the rise velocity is accelerated during the bubble ascension, the diminution of the film thickness brings about an acceleration of the mass transfer flux towards the bubble. In its turn, the accelerated volume increase leads to the faster augmentation of the rise velocity, a typically positive retroaction mechanism. Moreover, the thinning behaviour of the non-Newtonian fluids induces a diminution of the apparent viscosity, then a less drag for the bubble rise and a higher diffusion coefficient of $\mathrm{CO}_{2}$ in liquids (Li et al., 2001; Kemiha et al., 2006). This contributes simultaneously to the intensification of the mass transfer. The strange flow fields around a bubble rising in non-Newtonian fluids, in particular in PAAm solutions (Funfschilling \& Li, 2001), could also play a role in the mass transfer through a largely extended wake in space. In the face of such a tremendous complexity involving hydrodynamics, mass transfer, interfacial phenomena and rheology, a classical numerical approach is certainly beyond the reach. Based on our previous work of the Lattice Boltzmann (LB) applied to multiphase systems (Frank \& Li, 2005; Frank et al. 2006; Frank \& Li, 2006), we are currently trying to take up this challenge within the framework of the LB method.

\section{RESULTS AT MICROSCALE}

The recent development of advanced metrology such as the Particle Image Velocimetry (PIV) allows quantifying the flow features around bubbles in non-Newtonian fluids (Funfschilling \& Li, 2001; Li et al., 2001). By means of a newly acquired micro-PIV system (Dantec Dynamics), we tried to follow in time the nucleation and the growth process for a bubble attached to the nucleation site as well as the determination of the local flow field due to the expansion of bubble size in liquid within a microdevice similar to the above-mentioned macroscale setup. This $0.01 \times 0.01 \mathrm{~m}$ square visualisation chamber made of transparent PMMA (PolyMethylMethAcrylate) had a very thin depth 
$(0.001 \mathrm{~m})$. As it was still difficult to work with higher pressure, the $\mathrm{CO}_{2}$ saturation was realised under a relative pressure of 1 bar. Preliminary tests were carried out in demineralised water. The depressurisation was provoked by an opening of a manual valve.

This microdevice was horizontally loaded onto an inverted Leica microscope used as an for the micro-PIV. Thanks to this technique, it was possible to obtain the flow field of the continuous water phase with the help of seeding particles around a growing bubble after the depressurisation as well as the bubble size variation in time. The micro-PIV system was constituted of a Flowsense Dantec Camera with a $2048 \times 2048$ pixel array. The microscope was equipped with different objectives ranging from $\times 5$ to $\times 100$ ). The microdevice under investigation faced the microscope and was illuminated from the back by a microstrobe emitting at $530 \mathrm{~nm}$. Hydrophilic latex microspheres (Merck Estapor) with a density of $1056 \mathrm{~kg} \cdot \mathrm{m}^{-3}$ and a mean diameter of $0.88 \mu \mathrm{m}$ were used as seeding particles. These particles were small enough to follow the fluid and large enough to avoid the effects of Brownian motion.

The size growing in function of time is illustrated in Fig. 6 with a perfect spherical shape. Although the minimum nuclei size reported in this figure is limited to $15 \mu \mathrm{m}$, we expect to decrease much this size to $600 \mathrm{~nm}$ in further studies by means of a better illumination system. The bubble ascension was not observed in the microdevice due to its horizontal position. In Fig. 7, the flow fields were measured around two small bubbles of respectively 70 and $100 \mu \mathrm{m}$. It is worthy noting that the measurements of both nuclei size and flow fields at this scale were never reported yet in the literature to our best knowledge. In spite of some experimental perfection to gain, these original results can provide new insight into a theoretical modelling and numerical simulation at microscale that we envisage with the help of the LB approach. 


\section{CONCLUSION AND DISCUSSION}

The bubbles' nucleation and growth experiments were carried out in two transparent visualisation chambers at both macro- and microscale and under various operating conditions such as liquid's nature and initial supersaturation. The emphasis was given to the homogeneous nucleation in the bulk liquid. Two different mechanisms were identified as central to the nucleation and growth of a bubble: immobile at a stagnant nucleation site and an exponential increase for both the bubble diameter and rising distance after the detachment from the nucleation site.

For the stagnation nucleation at a site, the bubble growth has a first rapid period of about $2 \mathrm{~s}$ whatever the fluid's rheology. A linear increase following this fast period indicates the establishment of a permanent mass transfer regime before the detachment from the nucleation site. The first rapid increase could be mainly explained by both a high initial value of local supersaturation just after the depressurisation and a significant decrease of the surface tension force due to the bubble's size increase. However, accurate measurements of the supersaturation degree of dissolved $\mathrm{CO}_{2}$ gas in a non-Newtonian fluid are still required to go ahead for a possible modelling.

A simple physical reasoning based on the coupling between a force balance, rheology and experimental data of the of bubble size in function of time shows that it is logical to obtain an exponential increase of the rising distance with an exponential growing bubble size. Among the mass transfer efficiency, shear dependant viscosity, surface tension, bubble size and rise velocity, there is a positive retroaction mechanism. To a certain extent, a fractal scaling could be applied to this exponential variation for both the bubble size and rising distance. Clearly, the complex coupling nature of these different mechanisms cannot be handled yet by a classical Computational Fluid Dynamics (CFD) approach. In particular, physical understandings at microscale are seriously missing for the closure conditions at the interface between the bubble and surrounding fluid phase. 
To gain insight into this extraordinary complexity involving hydrodynamics, mass transfer, and interfacial phenomena, some preliminary results were obtained within a microdevice by means of a micro-PIV device Particle Image Velocimetry (PIV). The dynamical bubble growth at a nucleation site and corresponding flow fields around the bubble were quantified at microscale for the first time. We have shown the particular suitability of a Lattice Boltzmann (LB) approach for complex multiphase flows in our previous works. These new experimental results by the micro-PIV lead to foresee some possibilities to develop and validate a LB scheme for modelling and simulating the nucleation and growth of bubbles in fluids.

\section{NOTATION}

C gas concentration in liquid (mole. $\left.\mathrm{m}^{-3}\right)$

$C^{*} \quad$ gas concentration in liquid at the bubble interface $\left(\right.$ mole. $\left.\mathrm{m}^{-3}\right)$

$C_{d} \quad$ drag coefficient

$D \quad$ gas diffusion coefficient in liquid $\left(\mathrm{m}^{2} \cdot \mathrm{s}^{-1}\right)$

$d_{B} \quad$ bubble diameter $(\mathrm{m})$

$H \quad$ Henry's constant (J.mole $\left.{ }^{-1}\right)$

$K \quad$ fluid consistency $\left(\right.$ Pa. $\left.\mathrm{s}^{n}\right)$

$m \quad$ added mass $(\mathrm{kg})$

$n \quad$ flow index

$P \quad$ relative gas pressure $(\mathrm{Pa})$

$P_{0} \quad$ atmospheric pressure $(\mathrm{Pa})$

$P_{B} \quad$ pressure in the bubble $(\mathrm{Pa})$

$R \quad$ bubble radius (m)

$u \quad$ bubble rise velocity $\left(\mathrm{m} \cdot \mathrm{s}^{-1}\right)$ 
$V \quad$ bubble volume $\left(\mathrm{m}^{3}\right)$

Greek letters

$\delta \quad$ film thickness (m)

$\dot{\gamma} \quad$ shear rate $\left(\mathrm{s}^{-1}\right)$

$\varphi \quad$ mass transfer flux (mole. $\mathrm{s}^{-1}$ )

$\mu \quad$ generalised fluid viscosity (Pa.s)

$\rho_{L} \quad$ fluid density (kg.m $\left.\mathrm{m}^{-3}\right)$

$\rho_{G} \quad$ gas density $\left(\mathrm{kg} . \mathrm{m}^{-3}\right)$

$\sigma \quad$ surface tension (N.m ${ }^{-1}$ )

$\tau \quad$ stress $(\mathrm{Pa})$

\section{REFERENCES}

Abraham, F.F., (1974). Homogeneous nucleation theory, Academic Press, New York

Amon, M. \& Denson, C.D., A study of the dynamics of foam growth: analysis of the growth of closely spaced spherical bubbles, Polym. Eng. Sci., 24 (1984), 1026-1034

Barker, G.S., Jefferson, B. and Judd, S.J., The control of bubble size in carbonated beverages, Chem. Eng. Sci., 57 (2002), 565-573

Blander, M., (1975). Bubble nucleation in liquids, AIChE J., 21, 833-848

Chhabra, R.P. (1992). Bubbles, Drops \& Particles in Non-Newtonian Fluids. CRC Press, Boca Raton.

Frank, X. \& Li, H.Z., (2005). Complex flow around a bubble rising in a non-Newtonian fluid, Phys. Review E, 71, 036309

Frank, X., Funfschilling, D., Midoux, N. \& Li, H.Z., (2006). Bubbles in a viscous liquid: Lattice Boltzmann simulation and experimental validation, J. Fluid Mech., 546, 113-122 
Frank, X. \& Li, H.Z., (2006). Negative wake behind a sphere rising in viscoelastic fluids: a lattice Boltzmann investigation, Phys. Review E, 74, 056307

Funfschilling, D. \& Li, H.Z., (2001). Flow of non-Newtonian fluids around bubbles: PIV measurements and birefringence, Chem. Eng. Sci., 56, 1139-1141

Jones, S.F., Evans, G.M. and Galvin, K.P., (1999). Bubble nucleation from gas cavities - a review, Adv. Colloid Interface Sci., 80, 27-51

Kemiha, M., Frank, X., Poncin, S. and Li, H.Z., (2006). Origin of the negative wake behind a bubble rising in non-Newtonian fluids, Chem. Eng. Sci. 61, 4041-4047

Kungle, T.D. \& Beckman, E.L., (1983), Bubble dissolution physics and the treatment of decompression sickness, Medical Physics, 10, 184-190

Li, H.Z., Frank X., Funfschilling, D. \& Mouline, Y., (2001). Towards the understanding of bubble interactions and coalescence in non-Newtonian fluids: a cognitive approach. Chem. Eng. Sci., 56, $6419-6425$

Li, H.Z., Mouline, Y. and Midoux, N., (2002). Modelling the bubble formation dynamics in nonNewtonian fluids, Chem. Eng. Sci. 57, 339-346

Liger-Belair, G., Vignes-Adler, M., Voisin, C., Robillard, B. and Jeandet, P., Kinetics of gas discharging in a glass of champagne : The role of nucleation sites, Langmuir 18 (2002), 1294-1301 Milne-Thomson, L.M, (1960). Theoretical hydrodynamics, Macmillan, New York. Patel, R.D., (1980). Bubble growth in a viscous Newtonian liquid, Chem. Eng. Sci., 35, 2352-2356 Shafi, M.A. \& Flumerfelt, R.W., (1997). Initial bubble growth in polymer foam processes, Chem. Eng. Sci., 52, 627-633

Street, J.R., Fricke, A.L. and Reiss, L.P., (1971). Dynamics of phase growth in viscous nonNewtonian liquids, Ind. Eng. Chem. Fundam., 10, 54-64

Wienecke, M., Luke, A., Gorenflo, D. and Span, R., Flow boiling of highly viscous fluids in a vertical annular tube, Trans. IChemE., 83A (2005), 1044-1051 
Wu, J., Lu, Z.Y., Hu, J.C., Feng, L., Huang, J.D. \& Gu, X.S., (2006). Disruption of granules by hydrodynamic force in internal circulation anaerobic reactors, Wat. Sci. Tech., 54, 9-16

Xu, D., Pop-Iliev, R., Park, C.B. and Fenton, R.G., (2005). Fundamental study of CBA-blown bubble growth and collapse under atmospheric pressure, Journal of Cellular Plastics, 41, 519-538

Yang, C.T., Smith, T.G. \& Bigio, D.I., (1997). Polymer Trace Devolatilisation, AIChE J., 43, 18611883

Zhang, Y., (1998). Experimental simulations of gas-driven eruptions : kinetics of bubble growth and effect of geometry, Bull. Volcanol., 59, 281-290

Acknowledgment: The support from the Sino-French Advanced Research Program (PRA 15619RH) is greatly appreciated.

Figure captions

Figure 1. Transparent visualisation chamber for the bubble nucleation and growth.

Figure 2. Nucleation and growth of a $\mathrm{CO}_{2}$ bubble at a nucleation site in non-Newtonian fluids under various relative pressures.

Figure 3. Rising distance of a $\mathrm{CO}_{2}$ bubble after the detachment from a nucleation site. Time zero corresponds to the beginning of the departure.

Figure 4. After the detachment from a nucleation site, diameter increase of a $\mathrm{CO}_{2}$ bubble during the ascension (at the beginning of the departure, $t=0$ ). 
Figure 5. Comparison of the rising distance of a $\mathrm{CO}_{2}$ bubble between the model prediction and experimental measurement in $1 \% \mathrm{CMC}$ solution with an initial relative pressure $P=2$ bars.

Figure 6. $\mathrm{A} \mathrm{CO}_{2}$ bubble growth at a nucleation site in water under an initial relative pressure of 1 bar: visualisation by the micro-PIV device with 250 images. ${ }^{-1}$.

Figure 7. Flow field around a growing $\mathrm{CO}_{2}$ bubble at a nucleation site in water measured by the micro-PIV device with 1000 images. $\mathrm{s}^{-1}$ and a spatial resolution of $2048 \times 2048$. (a) $t=1.6 \mathrm{~s}$ and $d_{B}=$ $70 \mu \mathrm{m}$; (b) $t=4.8 \mathrm{~s}$ and $d_{B}=100 \mu \mathrm{m}$.

Table 1. Rheological properties of the fluids used.

\begin{tabular}{ccccc}
\hline & $1 \%$ CMC & $2 \%$ CMC & 0.5 PAAm & $0.5 \%$ Xanthan \\
\hline$K\left(\right.$ Pa. $\left.^{\mathrm{n}}\right)$ & 0.04 & 0.70 & 1.90 & 6.16 \\
\hline$n$ & 0.90 & 0.90 & 0.37 & 0.11 \\
\hline
\end{tabular}




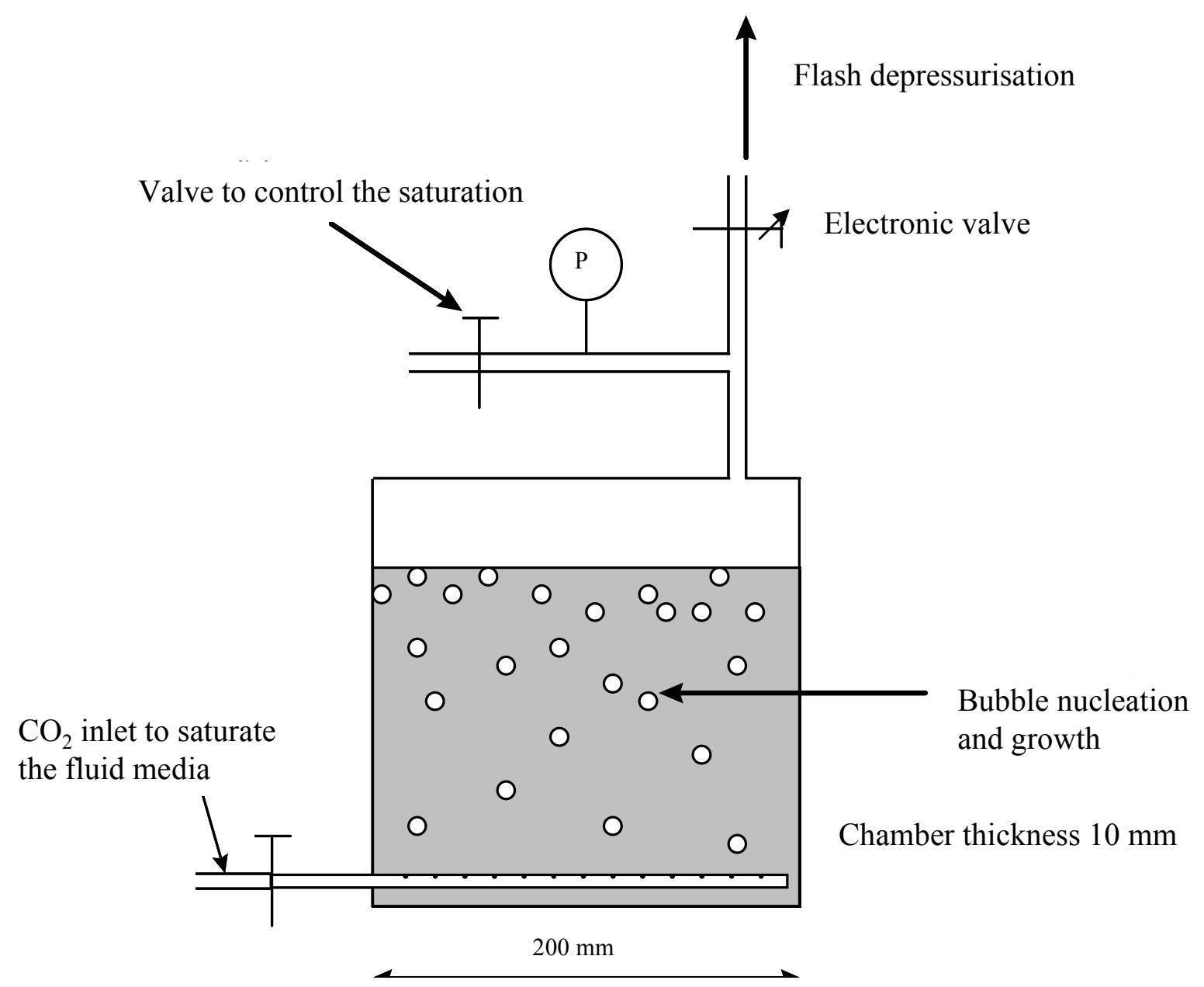

Figure 1. Transparent visualisation chamber for the bubble nucleation and growth. 


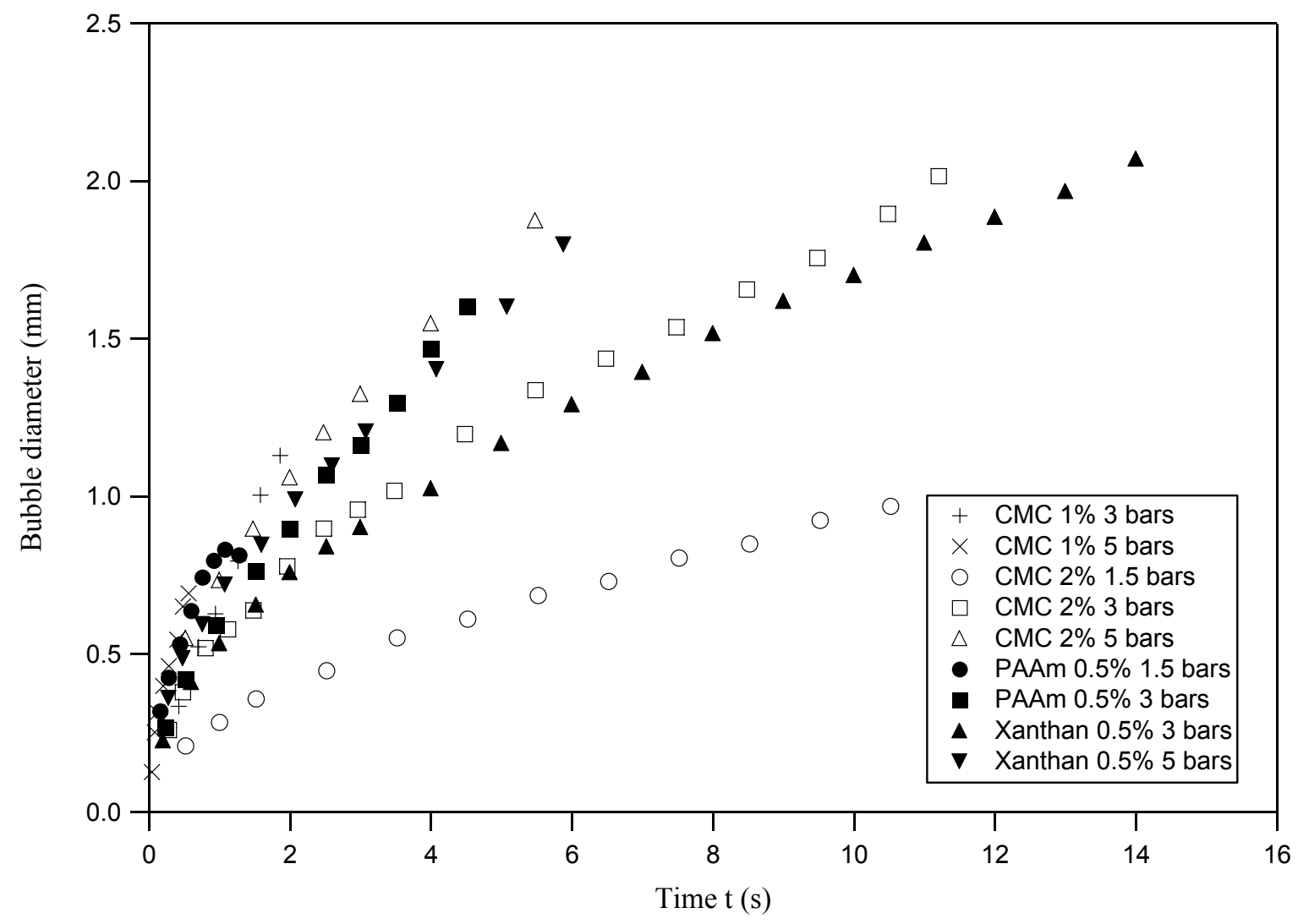

Figure 2. Nucleation and growth of a $\mathrm{CO}_{2}$ bubble at a nucleation site in non-Newtonian fluids under various relative pressures. 


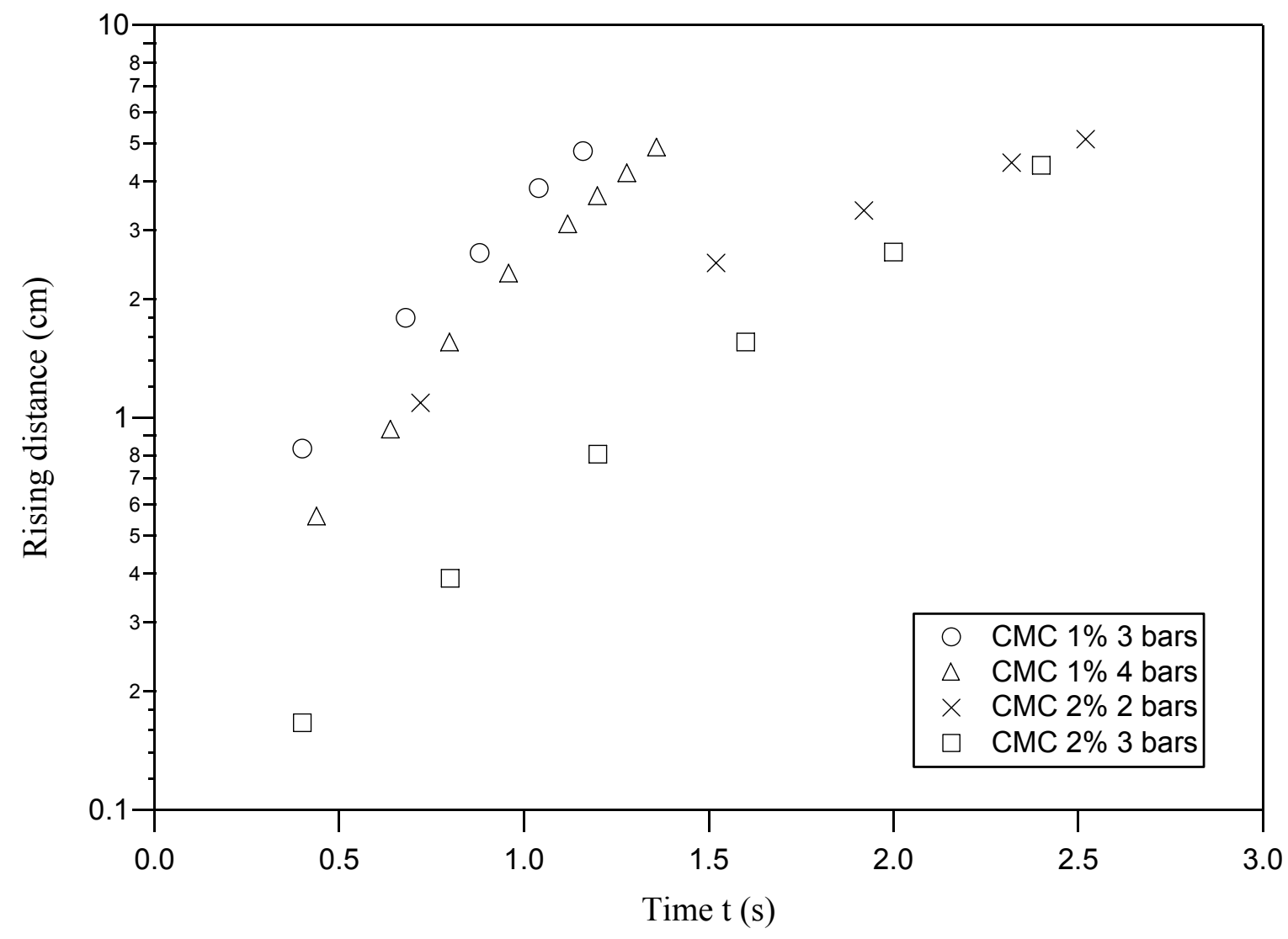

Figure 3. Rising distance of a $\mathrm{CO}_{2}$ bubble after the detachment from a nucleation site. Time zero corresponds to the beginning of the departure. 


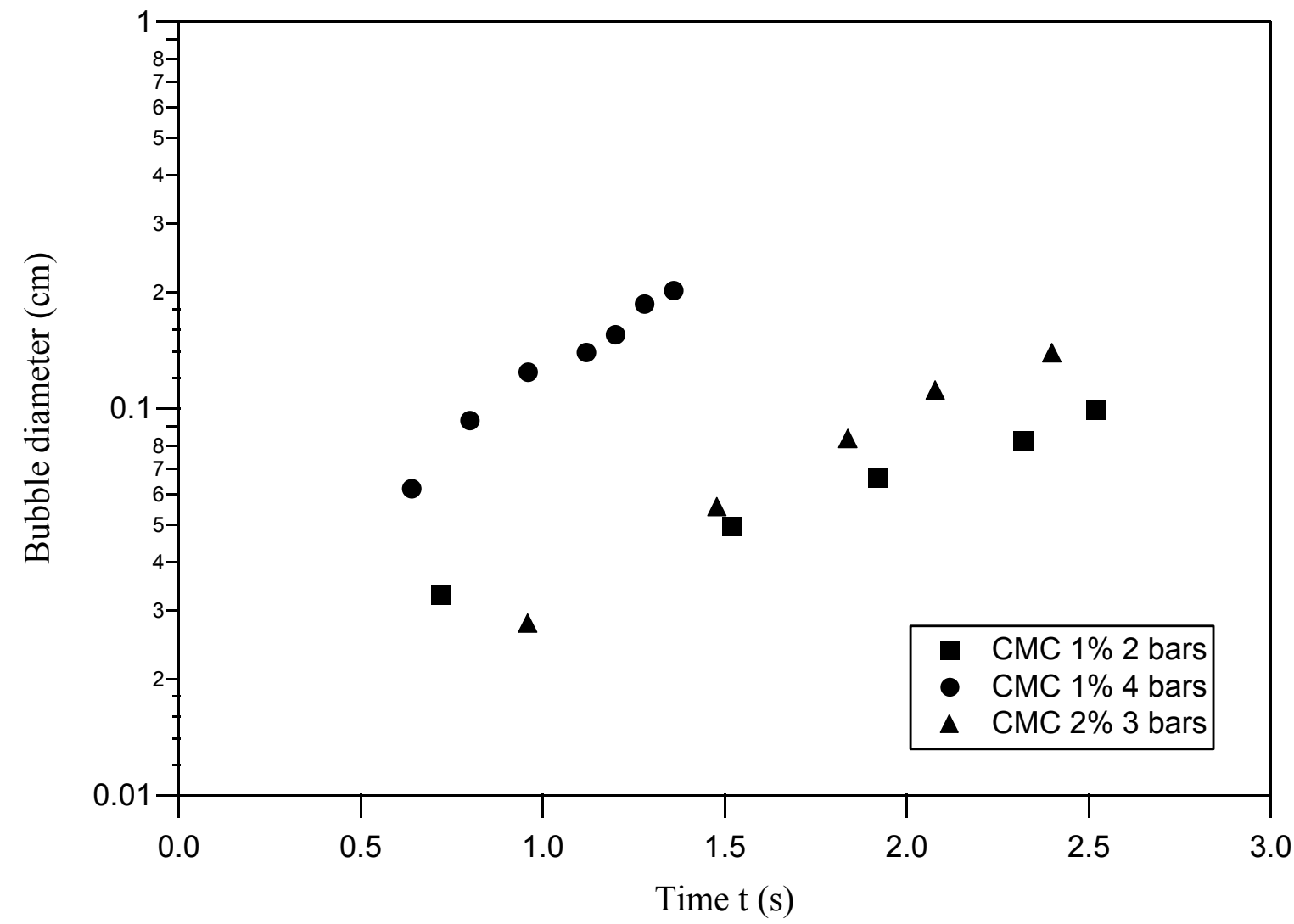

Figure 4. After the detachment from a nucleation site, diameter increase of a $\mathrm{CO}_{2}$ bubble during the ascension (at the beginning of the departure, $t$ $=0)$. 


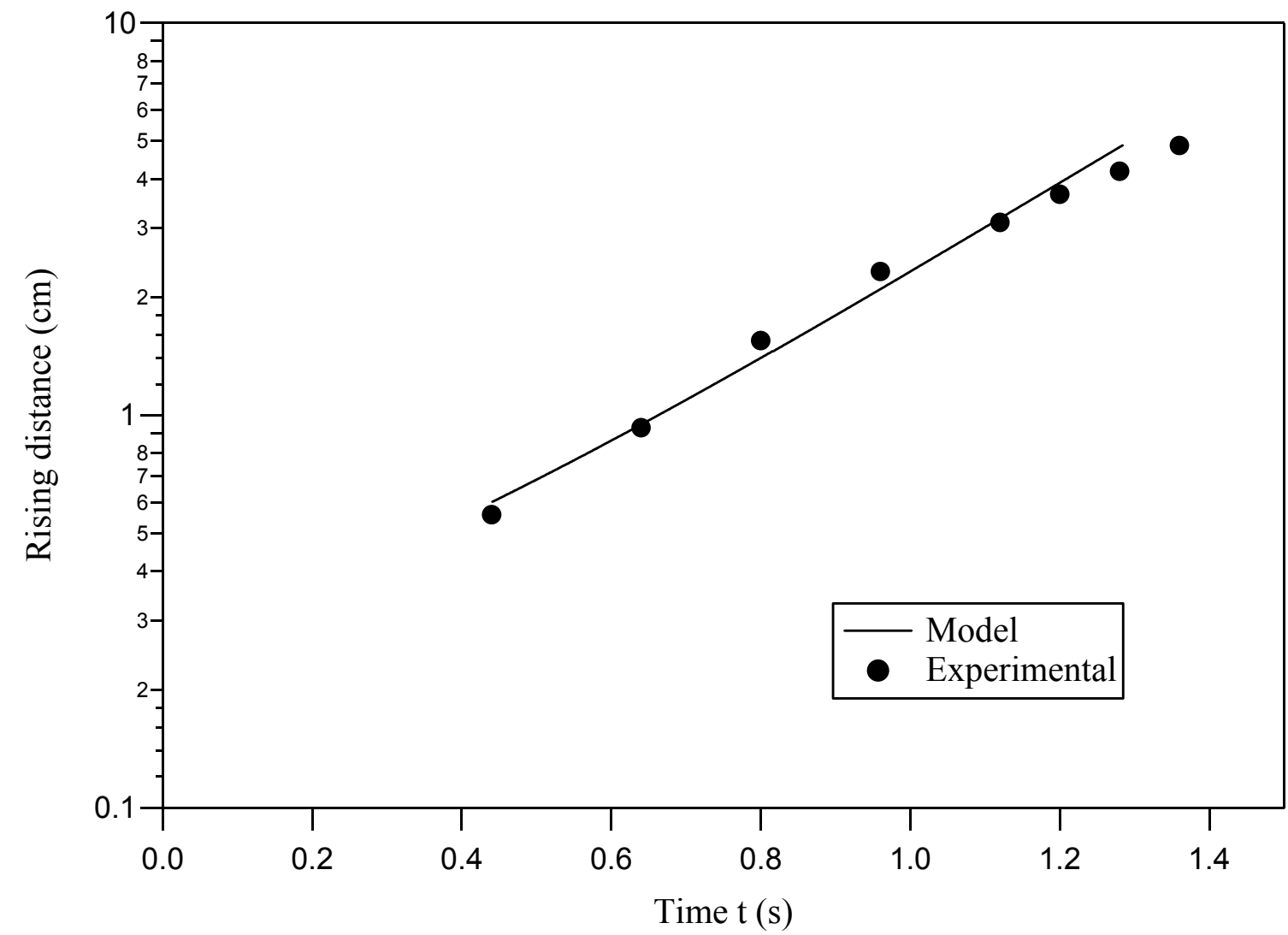

Figure 5. Comparison of the rising distance of a $\mathrm{CO}_{2}$ bubble between the model prediction and experimental measurement in $1 \% \mathrm{CMC}$ solution with an initial relative pressure $P=2$ bars. 


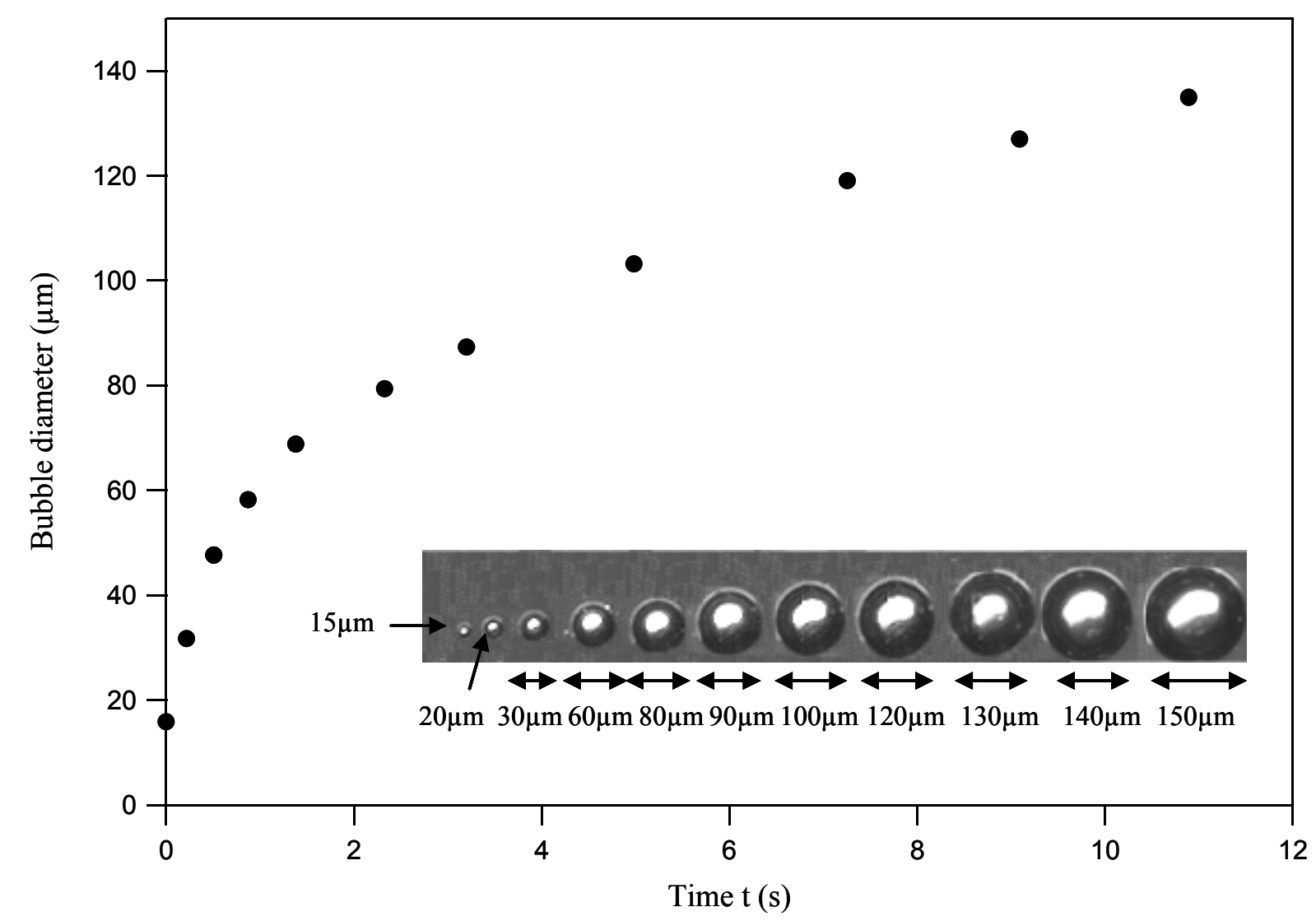

Figure 6. $\mathrm{A} \mathrm{CO}_{2}$ bubble growth at a nucleation site in water under an initial relative pressure of 1 bar: visualisation by the micro-PIV device with 250 images. $\mathrm{s}^{-1}$. 

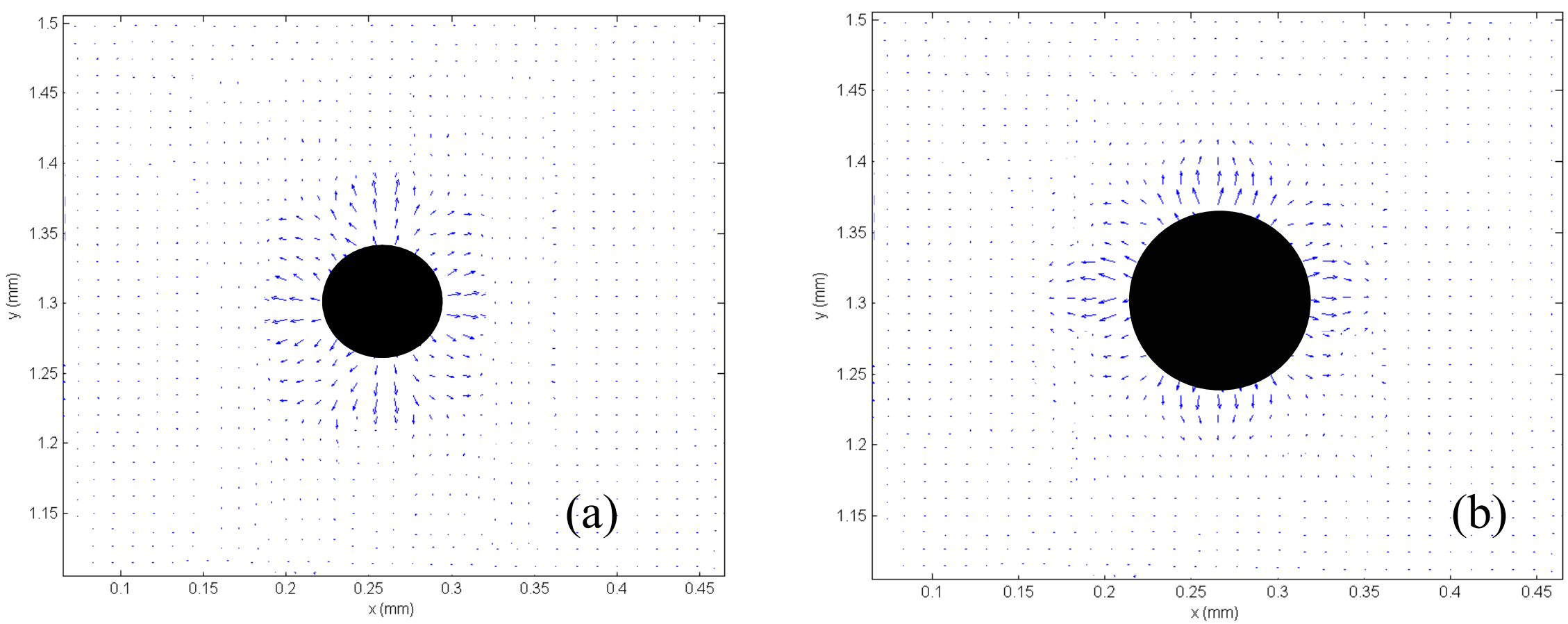

Figure 7. Flow field around a growing $\mathrm{CO}_{2}$ bubble at a nucleation site in water measured by the micro-PIV device with 1000 images.s ${ }^{-1}$ and a spatial resolution of $2048 \times 2048$. (a) $t=1.6 \mathrm{~s}$ and $d_{B}=70 \mu \mathrm{m}$; (b) $t=4.8 \mathrm{~s}$ and $d_{B}=100 \mu \mathrm{m}$. 